$(\mathrm{AOR}=2.37,95 \% \mathrm{CI}=1.26-2.93)$ each significantly related to increased odds of verbal violence. Short duration of employment (<five years) was the most strongly associated factor for sexual harassment $(\mathrm{AOR}=2.82$, 95\% $\mathrm{CI}=1.7-$ 2.94). Working the night shift was significantly associated with encountering verbal violence $(\mathrm{AOR}=2.79,95 \% \mathrm{CI}=$ 1.69-3.06).

Conclusion The findings of this study highlighted occupational violence and the need for an awareness of violence against female workers in industrial plants. The high prevalence of violence suggests that management in industrial workplaces should invest time and resources in addressing violence. Providing reporting procedure and training programs are recommended in dealing with workplace violence risk.

\section{0-158 LONG-TERM PROGNOSIS FOR HEALTH CARE UTILIZATION AMONG PATIENTS WITH WORK-RELATED MENTAL DISORDERS}

${ }^{1}$ Morten Vejs Willert, Marianne Kyndi, Vita Ligaya Dalgaard, David Høyrup Christiansen, Johan Hviid Andersen. 'Aarhus University Hospital, Denmark

\subsection{6/OEM-2021-EPI.114}

Objective The long-term prognosis for employees with workrelated stress, depression or PTSD is unclear. We aim to investigate if these conditions represent a temporary setback or a decline towards long-term poor health.

Methods From the Danish Occupational Medicine Cohort we included patients referred 2000-2012 with mental disorders $(\mathrm{N}=17.900)$ to Departments of Occupational Medicine across Denmark. From nation-wide registries we obtained data on general health care service utilization (GP contacts) and specialized mental health care (incident private practice psychiatrist/ psychologist care and psychiatric hospital care) for five years after/before the index-date of referral. Results are presented as utilization 5 years before referral (index-year -5), the year on either side of referral (index-year -1 and +1 ) and five years after referral (index-year +5 ).

Results General health care utilization increased from 7 median GP contacts in index-year -5 rising sharply to 12 in index-year -1 and 10 in index-year +1 , then declining gradually to 8 in index-year +5 . Incident private practice psychiatrist/psychologist care was $4.5 \%$ among patients in index-year -5 rising to $13.6 \%$ in index-year $-1,19.8 \%$ in index-year +1 , declining to $7.4 \%$ in index-year +5 . Incident psychiatric hospital care was $1.4 \%$ in index-year -5 rising to $5.6 \%$ in indexyear $-1,8.6 \%$ in index-year +1 , then declining to $4.7 \%$ in index-year +5 .

Conclusions General health care utilization was elevated in the years surrounding referral, then declined to levels comparable to five years prior to referral. From a relatively low incidence, mental health care utilization increased 4-6 fold in the years around referral, then declined to levels 2-3 fold higher than five years before referral. This could indicate that work-related mental disorders represent a temporary setback for most patients, but for some becomes a decline towards long-term poor mental health. Further analyses will seek to identify atrisk groups from demographic, occupational and health-related prognostic factors.

\section{0-189 MENTAL HEALTH IN CHILDHOOD AND ADOLESCENCE MATTERS: LINKING MENTAL HEALTH TRAJECTORIES WITH EMPLOYMENT OUTCOMES IN YOUNG ADULTHOOD}

${ }^{1}$ Marlene Amalie Magerl, Karin Veldman, Ute Bültmann, Iris Arends. ${ }^{1}$ Faculty of Medical Sciences, University Medical Center Groningen and University of Groningen, Netherlands

\subsection{6/OEM-2021-EPI.115}

Introduction Mental health problems cause a considerable burden of disease in adolescents, with potential negative consequences on employment later in life. Earlier, five trajectories of internalising and externalising problems (high-stable, moderate-high, decreasing, moderate-low, low-stable) among adolescents from 11 to 19 years were identified and a relationship with employment status at age 19 was found. However, at age 19 , many participants were still in education.

Objectives This study aims to examine associations of trajectory membership with having paid work and type of employment contract in young adults at age 26 .

Methods Fifteen-year follow-up data of the longitudinal TRacking Adolescents' Individual Lives Survey (TRAILS) cohort study $(\mathrm{N}=1711)$ were used. Logistic regression analyses were applied to examine associations of trajectory membership of internalising and externalising problems with having paid work and type of employment contract at age 26. The analyses were stepwise adjusted for sex, intelligence, parental education, family composition, physical health, negative life events and mental health comorbidity, all measured at age 11 .

Results For internalising problems, the high-stable (adjusted OR: 2.19; 95\% CI: 1.17-4.11) and moderate-high (adjusted OR: 1.75 ; 95\% CI: 1.14-2.69) trajectories were strongly associated with not having paid work, compared to the low-stable trajectory. No associations were found between the trajectories of externalising problems and having paid work. For both internalising and externalising trajectories, no associations were found between trajectory membership and having a permanent or temporary employment contract.

Conclusion This study expands current knowledge about the impact of internalising problems on employment of young adults. To prevent the effects of mental health problems on work, a life course perspective is needed taking into account the development of mental health problems in childhood and adolescence. In the transition from school to work, integrating youth- and occupational healthcare may be key to prevent negative employment outcomes due to internalising problems.

\section{0-211 OCCUPATIONAL DAYTIME LIGHT EXPOSURE AND RISK OF INITIATING ANTIDEPRESSANT TREATMENT AMONG MEN AND WOMEN: A NATIONAL FOLLOW-UP STUDY}

${ }^{1}$ Anne Vested, Vivi Schlünssen, Lex Burdorf, Esben Meulengracht Flachs, Stine Daugaard, Jens Peter Ellekilde Bonde, Susan Peters, Anne Helene Garde, Zara Ann Stokholm, Morten Vejs Willert, Jesper Medom Vestergaard, Henrik Albert Kolstad. 'Aarhus University, Denmark

\subsection{6/OEM-2021-EPI.116}

Introduction Higher winter depression prevalence at temperate latitudes has been attributed to low daylight levels. Daytime light exposure varies across jobs.

Objectives In a nationwide study, we examined occupational daytime light exposure levels and risk of initiating antidepressant treatment among men and women. 